\title{
Average AoI of Cached Status Updates for a Process Monitored by an Energy Harvesting Sensor
}

\author{
Nikolaos Pappas*, Zheng Chen ${ }^{\dagger}$, and Mohammad Hatami ${ }^{\ddagger}$ \\ *Dept. of Science and Technology, Linköping University, Norrköping Campus, Sweden. \\ ${ }^{\dagger}$ Dept. of Electrical Engineering, Linköping University, Linköping, Sweden. \\ ${ }^{\ddagger}$ Center for Wireless Communications, University of Oulu, Finland. \\ Email: \{nikolaos.pappas, zheng.chen\}@liu.se, mohammad.hatami@oulu.fi
}

\begin{abstract}
In this work, we consider a system where external requests arrive for status updates of a remote source, which is monitored by an energy harvesting (EH) sensor. The requests are placed in an aggregator that has direct communication with the sensor and is also equipped with storage space to cache a previously generated status update. A probabilistic model is considered to determine whether a new request will be served with a fresh update from the EH sensor or with a cached update from the aggregator. In the first case, the fresh update will replace the cached one in the aggregator. Assuming that the energy arrivals at the sensor can be modeled by a Bernoulli process, we characterize the average Age of Information (AoI) of the source seen at the aggregator as a function of the external request probability, the battery charging probability, and the probability that a fresh update will be generated by the EH sensor. Our numerical results reveal some insights about the role of caching in EH-based status updating systems.
\end{abstract}

Index Terms-Age of Information, Energy Harvesting, Caching.

\section{INTRODUCTION}

The Age of Information (AoI) was introduced recently as a metric and tool that captures the information freshness. It was first introduced in [1], [2]. This metric is particularly meaningful for some Internet of Things (IoT) applications that generate time-sensitive data. Such IoT applications consider the reception of status updates of some remote processes that evolve over time. Therefore, receiving fresh information, i.e., keeping the AoI small, is crucial. The notion of AoI has been extended to metrics such as the Value of Information, Cost of Update Delay, and non-linear AoI. A detailed treatment of this topic can be found in [3], [4].

The deployment of energy harvesting (EH) sensors is becoming a promising aspect of future wireless networks, especially in the IoT scenario where low-power devices transmit sporadically a small amount of information. Furthermore, EH is an enabling technology to create self-operating IoT networks with sensors in those areas that are hard to reach. The role of AoI and its effect in the efficient design of freshness-aware IoT networks was presented in [5].

If the sensor that generates the status updates by sampling/measuring a process is energy-limited, it cannot always be available to transmit. This issue can be resolved by having an intermediate node, an aggregator for example, with caching capabilities to store previously generated status updates by the sensor. The aggregator is responsible for forwarding the updates to the requesting devices.

\section{A. Related Works}

AoI for an energy harvesting source node has been investigated under various system settings. In [6]-[15], the EH process is modeled as an independent external stochastic process, usually as Bernoulli or Poisson process. In [12], the interplay of throughput/delay and average AoI was studied in a two-user multiple access channel consisting of an EH sensor and another node always connected to the power grid. The authors in [13] considered an EH status update system that monitors a stochastic process which can be either in a normal or in an alarm state of operation and the optimal status update transmission policies are derived. In [14], considers the average AoI minimization in cognitive radio $\mathrm{EH}$ communications. In [15], the minimization of the time-average expected AoI for status updates sent by an energy-harvesting source with a finite-capacity battery is considered.

Another set of works consider the case where a source is powered by radio frequency (RF) energy harvesting. In that case, the harvested energy depends on the channel condition and its variation over time. More specifically, the work in [16] studies the performance of a wireless powered sensor network in terms of the average AoI. The sensor node transmits updates to the destination by discharging its battery, which is charged by a dedicated energy source. The work in [17] considers freshness-aware IoT networks with EH enabled IoT devices. The optimal sampling policy for IoT devices that minimizes the long-term weighted sum-AoI is investigated. In [18], a cognitive network with primary and secondary users coexisting is considered. The secondary users harvest wireless energy from active primary ones and access the spectrum opportunistically to transmit status updates, then the average AoI is analyzed.

The works in [19]-[25] consider cache freshness. In [19], a system consisting of a library with time-varying files is considered where a server observes the current version of all files, and a cache that stores the current versions of all files and updates fresh versions of these files from the server. The update duration is considered to change depending on the file and its AoI. The authors in [20], propose a model for mobile caching where the rate of requests for content 
depends on the popularity and the freshness of the information. The work in [22] considers an updating policy for a system where a local cache is connected to a remote server through a capacity-constrained link and the cache maintains a collection of multiple content items that are requested by users. In [24], a cache refresh system is considered where a server is connected to multiple sources and stores local copies of the data items at the sources. The allocation of refresh rate at the server for each source is investigated to maintain overall data freshness given limited refresh rate.

\section{B. Contribution}

The main contribution of this work is about investigating the role of caching in a status updating system, where a sensor monitoring a remote source relies solely on EH to operate. An aggregator with caching capabilities serves as an intermediate node between the sensor and the external requests for status updates. The latest received status update is always stored in the cache of the aggregator. When a new request for status update arrives at the aggregator, with some probability it can either be served with a fresh update generated by the sensor or with a cached update in the aggregator. Based on this probabilistic model, we characterize the average AoI of the source at the aggregator which affects the freshness of the status updates that serve the external requests. The impact of different parameters on the average AoI is shown by numerical results.

\section{SySTEM MODEL}

In this work, we consider a discrete-time system depicted in Fig. 1, where an energy harvesting sensor $S$ can measure a process and send the measurement in a form of a status update to an aggregator $C$. $C$ can store the update and potentially utilize it when an external request for status update arrives. $S$ is harvesting energy from the environment, the energy arrival process is modeled as a Bernoulli process with average probability $\delta$. In addition, we assume $S$ is equipped with an infinite size battery ${ }^{1}$. We consider that the size of status update packets are the same, and the transmission of a status update from $C$ is instantaneous and error free. When $C$ requests for a fresh update from $S$, we assume that the whole duration of requesting, sampling, and transmission from $S$ occupies a slot and it consumes one energy unit from the battery of $S$.

An external request for status update arrives in a timeslot with probability $p$, and is served by either a cached update from $C$ or a fresh update from $S$ through $C$. If the battery at $S$ is non-empty, and there is an external request for update at $C$, then with probability $\alpha, C$ will request for a fresh update from $S$. Otherwise, $C$ will serve the external request with the cached update with probability $1-\alpha$. Furthermore, if $S$ has an empty battery, then $C$ will serve an external request with the cached update. Every time $C$ gets a fresh update from $S$, the update is stored in $C$ 's cache and the previously stored update is dropped since it becomes obsolete. If in a timeslot there is

\footnotetext{
${ }^{1}$ The extension to the finite size battery is straightforward [26, pp. 69].
}

no external request then $C$ will never ask for a fresh update from $S$.

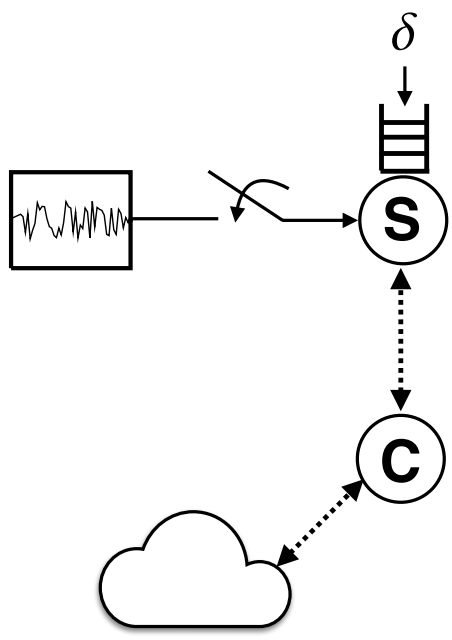

Figure 1. The system model. $C$ is the aggregator with cache, $S$ is the energy harvesting sensor who samples the source and creates the status updates. $\delta$ denotes the energy arrival probability.

\section{A. Analysis for the battery at $S$}

In this subsection we give more details regarding the operation of the battery at $S$ and we will provide the probability that the battery at $S$ is empty. We assume that the energy arrival process is Bernoulli with probability $\delta$. Furthermore, $C$ asks for a fresh update from $S$ with probability $\alpha$, when there is an external request and if the battery is not empty. Thus, one energy chunk will be consumed from the battery at $S$ with probability $p \alpha$. Then, the battery can be modeled by a Geo/Geo/1 queue with arrival probability $\delta$ and service probability $p \alpha$. We consider an early departure, late arrival model for the queue, thus we can obtain $\operatorname{Pr}(B=0)=1-\frac{\delta}{p \alpha}$ if $\delta<p \alpha$. If $\delta \geq p \alpha$, the Markov chain that models the $\mathrm{Geo} / \mathrm{Geo} / 1$ queue is not positive recurrent, however, this case can capture the scenario where $S$ is constantly connected to the power.

\section{Average AoI Analysis}

In this section, we provide the analysis regarding the average AoI that $C$ has about the source measured by $S$. At a time slot $t$, the AoI $\Delta(t)$, seen at $C$, is $\Delta(t)=t-G(t) . G(t)$ is the time the update stored at $C$ was generated at $S$. Since discrete time is assumed, the AoI values are in the set of natural numbers. A sample path for the AoI evolution can be found in Fig. 2.

$R_{i}$ denotes the time between two consecutive external requests, i.e., $i$-th and $(i+1)$-th; $T_{k}$ is the elapsed time between reception of $k$-th and the $(k+1)$-th fresh status updates from $S, N$ denotes the number of served external requests between the two fresh status updates. Then we have that

$$
T_{k}=\sum_{i=1}^{N} R_{i} .
$$




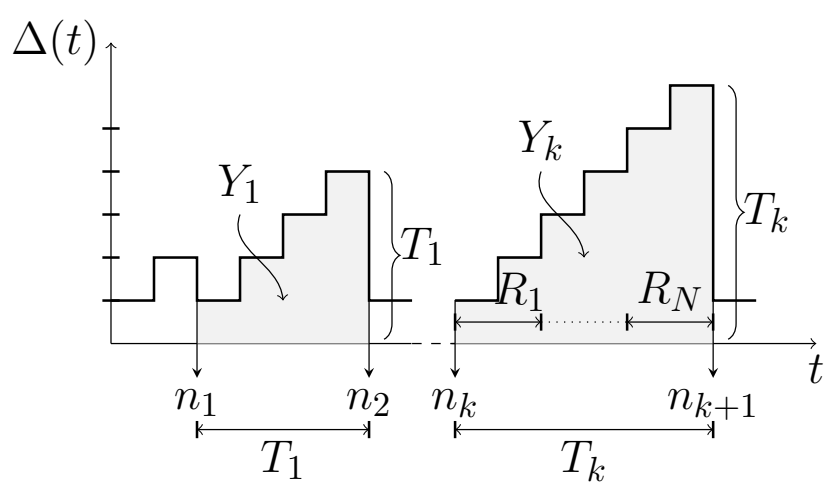

Figure 2. An example for the evolution of the AoI $\Delta(t)$. Every time that an external request is served with a fresh update, AoI becomes one, since the fresh update is stored in the cache of $C$.

Here $N$ is a random variable. Note that $T_{k}$ is a stationary process, thus, $\mathbb{E}[T]=\mathbb{E}\left[T_{k}\right]$ and $\mathbb{E}\left[T^{2}\right]=\mathbb{E}\left[T_{k}^{2}\right]$ for any $k$, in addition we have $\mathbb{E}[R]=\mathbb{E}\left[R_{k}\right]$, and $\mathbb{E}[R]=\frac{1}{p}$.

We consider a period of $M$ time slots where $U$ external requests for updates are placed and served. In order to derive the average AoI we proceed as follows

$$
\Delta_{M}=\frac{1}{M} \sum_{m=1}^{M} \Delta(m)=\frac{1}{M} \sum_{k=1}^{U} Y_{k}=\frac{U}{M} \frac{1}{U} \sum_{k=1}^{U} Y_{k}
$$

Since $\lim _{M \rightarrow \infty} \frac{U}{M}=\frac{1}{\mathbb{E}[T]}$, and $\frac{1}{U} \sum_{k=1}^{U} Y_{k}$ is the average of $Y$, we have

$$
\Delta=\lim _{N \rightarrow \infty} \Delta_{N}=\frac{\mathbb{E}[Y]}{\mathbb{E}[T]} .
$$

From Fig. 2, we obtain that

$$
Y_{k}=\sum_{m=1}^{T_{k}} m=\frac{T_{k}\left(T_{k}+1\right)}{2}
$$

Then we have

$$
\Delta_{M}=\frac{U}{M} \frac{1}{U} \sum_{k=1}^{U} Y_{k}=\frac{\mathbb{E}\left[\frac{T_{k}^{2}}{2}+\frac{T_{k}}{2}\right]}{\mathbb{E}[T]}=\frac{\mathbb{E}\left[T^{2}\right]}{2 \mathbb{E}[T]}+\frac{1}{2} .
$$

We calculate $\mathbb{E}[T]$ as follows

$$
\mathbb{E}[T]=\sum_{N=1}^{\infty} N p^{N} \mathbb{E}[R] \operatorname{Pr}(C)^{N-1} \operatorname{Pr}(\mathcal{S}),
$$

where $\mathcal{S}$ denotes the event that an external request will be served with a fresh update from $S$. $C$, denotes the event that a stored update will be utilized for the external request. Then we obtain that $\operatorname{Pr}(C)=1-\alpha \operatorname{Pr}(B \neq 0)=1-\frac{\delta}{p}$ and $\operatorname{Pr}(\mathcal{S})=\frac{\delta}{p}$. Then, we have

$$
\mathbb{E}[T]=\sum_{N=1}^{\infty} N p^{N} \frac{1}{p}\left(1-\frac{\delta}{p}\right)^{N-1} \frac{\delta}{p}=\frac{\delta}{p} \sum_{N=1}^{\infty} N(p-\delta)^{N-1} .
$$

By utilizing that $\sum_{N=1}^{\infty} N r^{N-1}=\frac{1}{(1-r)^{2}}$ if $r<1$, we have that

$$
\mathbb{E}[T]=\frac{\delta}{p(1-p+\delta)^{2}},
$$

given that $\delta<p$, as we discussed in Section II-A.

For the second moment of $T$, we utilize that

$$
T_{k}^{2}=\sum_{i=1}^{N} R_{i}^{2}+\sum_{i=1}^{N} \sum_{j=1, j \neq i}^{N} R_{i} R_{j}
$$

Due to the stationarity of $R_{i}$, we use $\mathbb{E}[R]$ for the average of $R_{i}$ then, taking the conditional expectation of both sides, we obtain

$$
\mathbb{E}\left[T^{2} \mid N\right]=N \mathbb{E}\left[R^{2}\right]+N(N-1)(\mathbb{E}[R])^{2} .
$$

We calculate $\mathbb{E}\left[R^{2}\right]$ as follows

$\mathbb{E}\left[R^{2}\right]=\sum_{k=1}^{\infty} k^{2} \operatorname{Pr}(R=k)=\sum_{k=1}^{\infty} k^{2}(1-p)^{k-1} p=\frac{2-p}{p^{3}}$.

Note that $\sum_{k=1}^{\infty} k^{2} r^{k-1}=\frac{r+1}{(1-r)^{3}}$ if $r<1$. Then we have that

$$
\mathbb{E}\left[T^{2} \mid N\right]=N \frac{2-p}{p^{3}}+\frac{N(N-1)}{p^{2}} .
$$

Now we proceed with the calculation of $\mathbb{E}\left[T^{2}\right]$ as follows

$$
\begin{aligned}
\mathbb{E}\left[T^{2}\right] & =\sum_{N=1}^{\infty} \mathbb{E}\left[T^{2} \mid N\right] p^{N} \mathbb{E}[R] \operatorname{Pr}(C)^{N-1} \operatorname{Pr}(\mathcal{S}) \\
& =\frac{\delta}{p} \sum_{N=1}^{\infty} \mathbb{E}\left[T^{2} \mid N\right](p-\delta)^{N-1} \\
& =\frac{\delta(2-p)}{p^{4}} \sum_{N=1}^{\infty} N(p-\delta)^{N-1}+ \\
& +\frac{\delta}{p^{3}} \sum_{N=1}^{\infty} N(N-1)(p-\delta)^{N-1} \\
& =\frac{\delta(2-p)}{p^{4}(1-p+\delta)^{2}}+\frac{\delta}{p^{3}} \frac{2(p-\delta)}{(1-p+\delta)^{3}} .
\end{aligned}
$$

After substituting (8) and (13) into (5), we obtain that

$$
\Delta=\frac{2-p}{2 p^{3}}+\frac{p-\delta}{p^{2}(1-p+\delta)}+\frac{1}{2}
$$

\section{A. The case where $\delta>p \alpha$}

In this case, the battery is charging faster than discharging. Thus, we have that $\operatorname{Pr}(B \neq 0)=1$. Then, by following similar methodology as above, we obtain that

$$
\mathbb{E}[T]=\frac{\alpha}{(1-p(1-\alpha))^{2}},
$$

and

$$
\mathbb{E}\left[T^{2}\right]=\frac{\alpha(2-p)}{p^{3}(1-p(1-\alpha))^{2}}+\frac{2 \alpha(1-\alpha)}{p^{2}(1-p(1-\alpha))^{3}} .
$$

Thus, the average AoI in this case is 


$$
\Delta=\frac{2-p}{2 p^{3}}+\frac{1-\alpha}{p(1-p(1-\alpha))}+\frac{1}{2} .
$$

Remark 1. Note that the expression of the average AoI depends on the range of $p, \delta$, and $\alpha$. Even though the expression in (14) does not depend on $\alpha$, the values of $p$ and $\delta$ are constrained from the condition $\delta<p \alpha$.

Remark 2. The expression for the average AoI for the system without energy limitations at $S$, is given by (17) where $\alpha$ and $p$ vary independently.

\section{Numerical Results}

In this section, we present numerical results for the average AoI and investigate the impact of different parameters on it.

In Fig. 3, we illustrate the average AoI, $\Delta$, as a function of the external request probability $p$ for $\alpha=0.5$ and $\delta=\{0.02,0.05,0.1,0.2\}$. For comparison, we also depict the average AoI for the system without energy constraints, labeled as Non-EH.

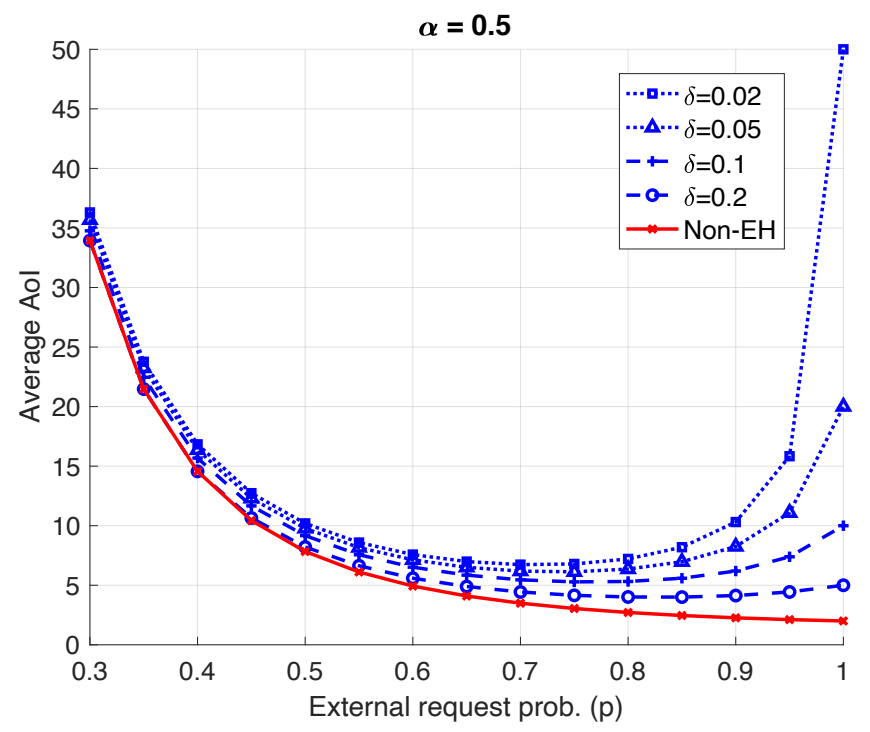

Figure 3. Average AoI vs $p$ for $\alpha=0.5$.

We observe that for the case without energy constraints, $\Delta$ is decreasing with $p$. Furthermore, with lower values of $p$, we observe that the performance is almost the same, or very close, among the different cases. This is expected, since the external requests are not stressing the battery of the sensor. However, when $p$ increases, we observe that $\Delta$ initially decreases and then increases, this behavior is more profound for $\delta=0.02$ and $\delta=0.05$, which is due to the fact that at some point the requests to the sensor for fresh updates are draining the battery. When $\delta=0.2$, we observe a similar performance trend to the Non-EH case, except a slight increase of $\Delta$ for $p>0.9$.

In Fig. 4 we present the average AoI versus $\alpha$ for $p=$ $\{0.4,0.8\}$ and $\delta=\{0.1,0.2\}$. For both values of $p$ we depict the performance for the system without energy constraints for comparison. We observe that as $\alpha$ increases, then $C$ is requesting more frequent fresh updates from $S$ given that it

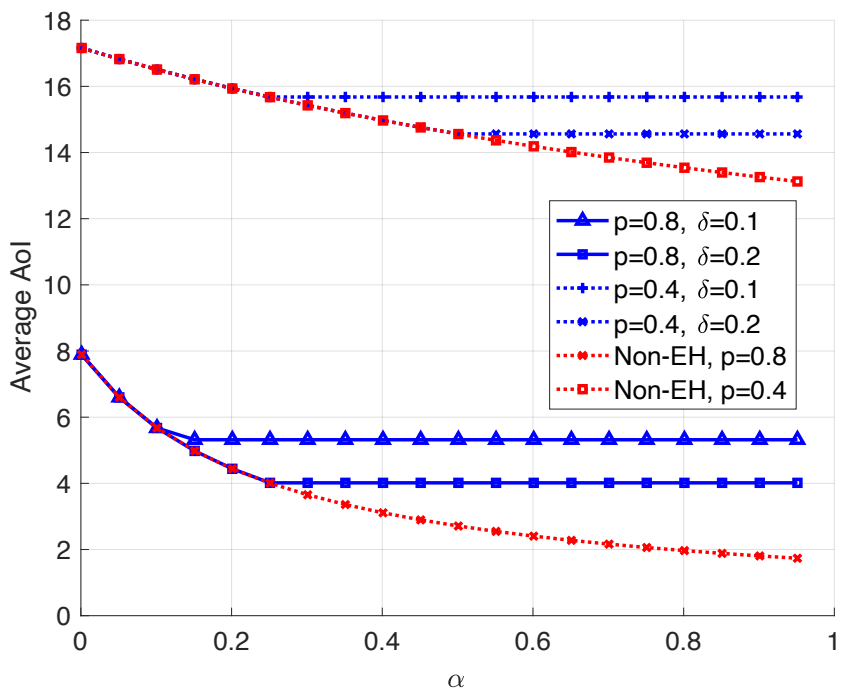

Figure 4. Average AoI vs $\alpha$.

has a non-empty battery. Note that when $\delta>p \alpha$ the the performance is close to performance of the system without energy constraints as also explained in Remark 2 in the previous section. In addition, for larger $p, \Delta$ decreases faster with $\alpha$.

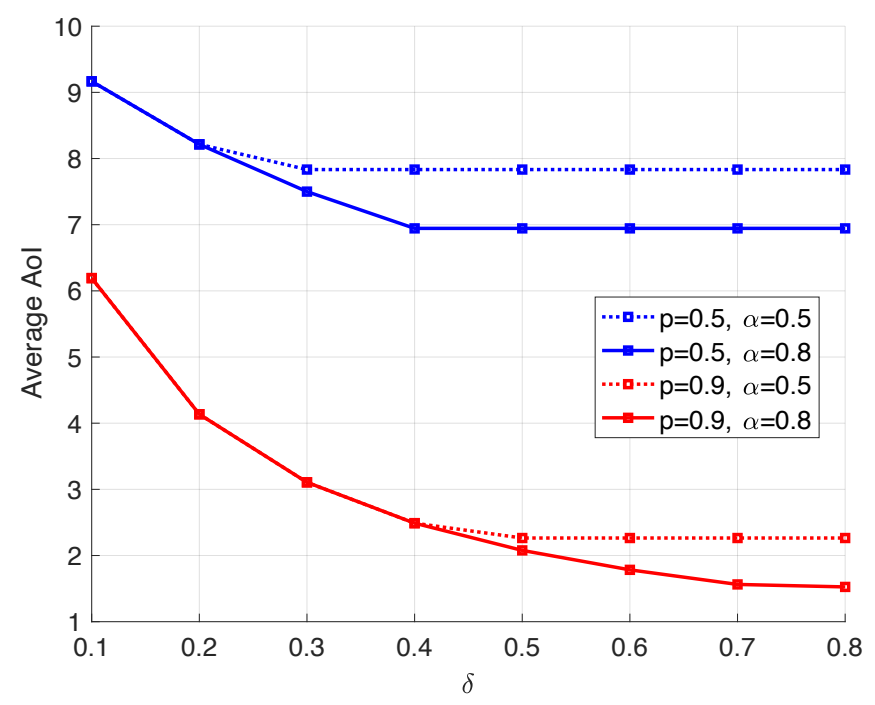

Figure 5. Average AoI vs $\delta$.

In Fig. 5, the average AoI versus $\delta$ is illustrated. We consider the cases with $p=\{0.5,0.9\}$ and $\alpha=\{0.5,0.8\}$. Note that the lowest $\Delta$ is achieved by the case $p=0.9$ and $\alpha=0.8$. However, for the lower values of $\delta$, the performance between $p=0.9, \alpha=0.8$ and $p=0.9, \alpha=0.5$ is the same, since as we saw in Section III, for $\delta<p \alpha, \Delta$ does not depend on $\alpha$ directly.

\section{Summary AND Future Directions}

In this work, we investigated the average AoI in a cacheenabled status updating system with an EH sensor that mon- 
itors a process. An external request for status update can be fulfilled by either receiving a fresh update generated by the $\mathrm{EH}$ sensor or a previously cached update at an aggregator, based on a probabilistic rule. The average AoI has been derived as a function of different system parameters. Our results showed how the energy arriving process and the status updating rule affect the information freshness in such system.

Future directions of this work include the case of multiple sensors, introducing errors in the transmissions, and delay in the sampling process.

\section{ACKNOWLEDGMENT}

This work was supported in part by the Center for Industrial Information Technology (CENIIT), ELLIIT, the Swedish Foundation for Strategic Research, and the Swedish Research Council (VR). The reserach of Mr. Hatami has been financially supported by the Infotech Oulu, the Academy of Finland (grant 323698), and Academy of Finland 6Genesis Flagship (grant 318927). The authors would like to thank Prof. Marian Codreanu for the fruitful discussions.

\section{REFERENCES}

[1] S. Kaul, M. Gruteser, V. Rai, and J. Kenney, "Minimizing age of information in vehicular networks," in 8th Annual IEEE Commun. Society Conf. on Sensor, Mesh and Ad Hoc Commun. and Networks, June 2011, pp. 350-358.

[2] S. Kaul, R. Yates, and M. Gruteser, "Real-time status: How often should one update?" in IEEE Conf. on Computer Commun. (INFOCOM), Mar. 2012, pp. 2731-2735.

[3] A. Kosta, N. Pappas, and V. Angelakis, "Age of information: A new concept, metric, and tool," Foundations and Trends ${ }^{\circledR}$ in Networking, vol. 12, no. 3, pp. 162-259, 2017.

[4] Y. Sun, I. Kadota, R. Talak, and E. Modiano, "Age of information: A new metric for information freshness," Synthesis Lectures on Communication Networks, vol. 12, no. 2, pp. 1-224, 2019.

[5] M. A. Abd-Elmagid, N. Pappas, and H. S. Dhillon, "On the role of age of information in the internet of things," IEEE Communications Magazine, vol. 57, no. 12, pp. 72-77, December 2019.

[6] R. D. Yates, "Lazy is timely: Status updates by an energy harvesting source," in IEEE Intl. Symposium on Information Theory (ISIT), June 2015, pp. 3008-3012.

[7] S. Farazi, A. G. Klein, and D. R. Brown, "Average age of information for status update systems with an energy harvesting server," in IEEE Conf. on Computer Commun. Workshops (INFOCOM WKSHPS), Apr. 2018, pp. 112-117.

[8] X. Wu, J. Yang, and J. Wu, "Optimal status updating to minimize age of information with an energy harvesting source," in IEEE Intl. Conf. on Commun. (ICC), May 2017, pp. 1-6.

[9] B. T. Bacinoglu and E. Uysal-Biyikoglu, "Scheduling status updates to minimize age of information with an energy harvesting sensor," in IEEE Intl. Symposium on Information Theory (ISIT), June 2017, pp. $1122-1126$.

[10] A. Arafa and S. Ulukus, "Age-minimal transmission in energy harvesting two-hop networks," in IEEE Global Commun. Conf. (GLOBECOM), Dec. 2017, pp. 1-6.

[11] _ _ "Age minimization in energy harvesting communications: Energycontrolled delays," in 51st Asilomar Conf. on Signals, Systems, and Computers, Oct. 2017, pp. 1801-1805.

[12] Z. Chen, N. Pappas, E. Björnson, and E. G. Larsson, "Age of information in a multiple access channel with heterogeneous traffic and an energy harvesting node," in IEEE INFOCOM - IEEE Conference on Computer Communications Workshops (INFOCOM WKSHPS), April 2019, pp. 662-667.

[13] G. Stamatakis, N. Pappas, and A. Traganitis, "Control of status updates for energy harvesting devices that monitor processes with alarms," in IEEE Global Commun. Conf. Workshops (GLOBECOM), Dec. 2019.
[14] S. Leng and A. Yener, "Minimizing age of information for an energy harvesting cognitive radio," in IEEE Wireless Communications and Networking Conference (WCNC), April 2019, pp. 1-6.

[15] B. T. Bacinoglu, Y. Sun, E. Uysal-Biyikoglu, and V. Mutlu, "Achieving the age-energy tradeoff with a finite-battery energy harvesting source," in IEEE Intl. Symposium on Information Theory (ISIT), June 2018, pp. 876-880.

[16] I. Krikidis, "Average age of information in wireless powered sensor networks," IEEE Wireless Communications Letters, vol. 8, no. 2, pp. 628-631, April 2019.

[17] M. A. Abd-Elmagid, H. S. Dhillon, and N. Pappas, "Online age-minimal sampling policy for rf-powered iot networks," in IEEE Global Commun. Conf. (GLOBECOM), Dec. 2019

[18] X. N. Shiyang Leng and A. Yener, "Age of information for wireless energy harvesting secondary users in cognitive radio networks," in IEEE International Conference on Mobile Ad Hoc and Sensor Systems (MASS), Nov 2019.

[19] H. Tang, P. Ciblat, J. Wang, M. Wigger, and R. Yates, "Age of information aware cache updating with file- and age-dependent update durations," 2019.

[20] C. Kam, S. Kompella, G. D. Nguyen, J. E. Wieselthier, and A. Ephremides, "Information freshness and popularity in mobile caching," in IEEE International Symposium on Information Theory (ISIT), June 2017, pp. 136-140.

[21] S. Zhang, J. Li, H. Luo, J. Gao, L. Zhao, and X. S. Shen, "Towards fresh and low-latency content delivery in vehicular networks: An edge caching aspect," in 10th International Conference on Wireless Communications and Signal Processing (WCSP), Oct 2018, pp. 1-6.

[22] R. D. Yates, P. Ciblat, A. Yener, and M. Wigger, "Age-optimal constrained cache updating," in IEEE International Symposium on Information Theory (ISIT), June 2017, pp. 141-145.

[23] W. Gao, G. Cao, M. Srivatsa, and A. Iyengar, "Distributed maintenance of cache freshness in opportunistic mobile networks," in IEEE International Conference on Distributed Computing Systems, June 2012, pp. $132-141$.

[24] J. Zhong, R. D. Yates, and E. Soljanin, "Two freshness metrics for local cache refresh," in IEEE International Symposium on Information Theory (ISIT), June 2018, pp. 1924-1928.

[25] M. Hatami, M. Leinonen, and M. Codreanu, "Online caching policy with user preferences and time-dependent requests: A reinforcement learning approach," in Proc. Asilomar Conference on Signals, Systems and Computers, Nov. 2019.

[26] R. Srikant and L. Ying, Communication networks: an optimization, control, and stochastic networks perspective. Cambridge University Press, 2013. 